\title{
Computing price trends in sequential auctions*
}

\author{
Olivier Chanel** \\ GREQAM-IDEP, Centre National de la Recherche Scientifique \\ Stéphanie Vincent*** \\ Danish Transport Research Institute
}

\section{Introduction}

The focus on multiple unit auctions over the last fifteen years has partly been motivated by the desire to explain the price-decline anomaly, a situation where objects are sold at lower prices than identical objects sold earlier during the same auction. Ashenfelter's (1989) finding of a price decline on wine auction data was the starting point for a new development in the theory of multiple unit auctions. ${ }^{1}$

Price trends raise many issues, since the expected revenue of the sellers and/or gains for the buyers may depend on the order in which the objects are sold (for sellers) or on the right time to bid (for buyers). In this case, the sellers sometimes negotiate with the auctioneer to obtain the best position for the object in the sale: the auctioneer tries to maximize the revenue generated by the sale, while the sellers try to maximize the expected sale price(s) of their object(s).

Since Ashenfelter (1989), many empirical studies have found evidence of declining prices in auctions of wine (McAfee and Vincent (1993), Di Vittorio and Ginsburgh (1994) and Ginsburgh (1998)), condominium units

* The authors are very grateful to Victor Ginsburgh, Alan Kirman. Michel Lubrano, Peter Lyk-Jensen, Stephen Martin, Costin Protopopescu and two anonymous referees for their helpful comments and suggestions. The usual caveat applies.

** Corresponding author: CNAS-GREQAM-IDEP, 2 rue de la CharitE, F-13002 Marseilles, France, e-mail : chanel@ehess.univ-mrs.fr.

*** Danish Transport Research Institute, Knuth.Winterfeldts AllĖ, Bygning 116 Vest, DK-2800 Kgs Lyngby, Denmark, e-mail : svl@dtf.dk.

1 Although Buccola (1982) found statistical evidence of a price decline in cattle auctions by using hedonic regressions. 
(Ashenfelter and Genesove (1992)), stamps (Taylor (1991)), commercial properties (Lusht (1994)), jewelry (Chanel et al. (1996)), works of art (Pesando and Shum (1996), Beggs and Graddy (1997)), flowers (Van den Berg et al. (2001)), fish (Pezanis-Christou (1997)) and so forth.

At first glance this price decline appears difficult to explain, because theory shows that sequential auctions of identical objects should result on average in identical or rising prices. These results come from Weber (1983), where prices for identical objects follow a random walk in the case of independent private values and display an upward drift in the case of general affiliated values. ${ }^{2}$ Because of this contradiction between the theory and empirical data, declining prices are considered an anomaly. Part of the debate in the literature concerns whether or not such an anomaly exists, i.e. whether or not declining prices can be explained by certain market characteristics and hence by rational bidders' behavior (see Fevrier (2003), for a recent overview of such theoretical explanations). The purpose of this paper, however, is to consider how price decline is computed in empirical studies. Its novelty consists in checking whether the method itself might not partly explain the phenomenon, and it shows the need for caution when interpreting trends measured in empirical studies.

Measuring price trends in a dynamic world is neither an easy task nor a neutral exercise. It is sufficient to recall the impassioned debates when the Boskin Commission (1996), looking for a "more accurate measure of the Cost of Living", concluded that the US consumer price index had an upward bias of 1.1 percentage points per annum. Since pensions, wages, taxes and transfers are generally index-linked to inflation, the existence of a bias has obvious implications for the level of public debt.

The methods used in auction literature to compute price trends require pairs of prices, and therefore, how a pair of lots in a parcel (a parcel is a set of identical objects or lots of objects sold sequentially) is selected worth investigating. ${ }^{3}$ The main findings of the paper are the following: the choice of the pairs of prices in a parcel is crucial ; the size of the parcels and the position of the lots in each parcel can influence the trend ; the methods used to measure the price trend may lead to significantly different results when used on the same sample, and even worse, the same method can give opposite results depending on the criterion used for selecting pairs.

The paper is structured as follows. Section 2 introduces the main price indices aggregating individual price variations and examines their properties and their spread. Section 3 tests these indices on wine auction data. Several hypotheses are tested using bootstrap techniques. Section 4 concludes the paper.

\footnotetext{
2 See also Milgrom and Weber (2000).

3 Most of the empirical papers study price trends between two objects or two lots of objects. However the lots usually belong to a parcel of more than two identical lots.
} 


\section{$2 \quad$ Measuring the trend}

For sequential auctions of multiple lots sold successively, the sequence of lots can be viewed as a sub-sale within the sale. The aim is to measure the trend of this sequence.

\subsection{Aggregating price variations of similar objects}

Price variations have to be aggregated in order to compute an overall price trend. This exercise is more complex than it sounds.

First, the objects are not fully identical. They can often be differentiated : quality of colors or preservation can differ between two prints of the same work; specific characteristics can explain a price difference between two bottles of the same wine (the "fullness" of a bottle, the color of the wine, the state of the cork, etc.). ${ }^{4}$ Information about the quality of the objects is lost unless one attends the auction and/or the pre-sale exhibition. Buyers may then take this information into account when bidding. If the objects (although similar) are ranked and auctioned according to their quality, the trend is likely to be downward biased. 5

Second, quantifying the price trend means aggregating price variations for parcels of similar goods. In effect, apart from the (hypothetical) case where the same trend (increase, decrease or stability) exists for each of the parcels, it is necessary to find a statistic that aggregates these variations, and to interpret it. Auction results show that it is possible to observe a $50 \%$ price variation between the prices of two similar objects sold during the same sale. Moreover, price levels of different parcels may exhibit a hundredfold variation! It is therefore important to verify whether the choice of aggregation mechanism can influence the resulting price trends.

Third, when there are more than two lots, some authors (Ashenfelter (1989), McAfee and Vincent (1993)) arbitrarily choose a pair of lots. Section 3 explores the consequences of such a choice on the valuation of the trend.

In this paper, we deliberately consider the lots within a parcel as identical, as our purpose is to study the influence of the overall methodology (the choice of the aggregation method and the selection criteria within the lots) on the resulting trends, and not to explain the trend observed. Consequently, we use the index number theory to compare the methods usually used to measure price trends in sequential auctions. Such numbers (for example Laspeyres, Paasche, Fisher, Walsch or Törnqvist indices) are widely used for constructing price indices by aggregating the variations observed between two periods (bilateral index) or by chaining them in order to study longer periods (multilateral index).

4 See Di Vittorio and Ginsburgh (1994) for more details.

5 Pesando and Shum (1996) mention for instance that Christiees and Sothebyes admit that when they sell two prints of the same work during the same sale, the better quality print always appears irst. 


\subsection{Two frequently used indices and their drawbacks}

Consider $N$ parcels $i, i=1, \ldots, N$ of similar lots of objects sold at auction. $P_{i}^{n}$ denotes the price of a lot sold at rank $n, n=1, \ldots, \bar{n}_{i}$, in parcel $i ; \bar{n}_{i}$ is the number of lots in parcel $i$, with $\bar{n}_{i} \geqslant 2 \forall i$.

In this section, only pairs of similar lots are explored $\left(\bar{n}_{i}=2\right)$. Section 3 will consider different ways of selecting pairs when there are more than two similar lots in a parcel $\left(\tilde{n}_{i}>2\right)$. Let $P_{i}^{1}$ and $P_{i}^{2}$ denote the prices of the two lots. Denote the ratio $P_{i}^{2} / P_{i}^{1}$ as $a_{i}$. Two types of price index are frequently used for sequential auctions of multiple objects : the Arithmetic Mean of Ratios (AMR) and the Ratio of the Sums of Prices (RSP).

The Arithmetic Mean of Ratios, certainly the most widely used in auction literature, consists in computing the ratio of the second price to the first price for each pair of objects, and then computing their arithmetic mean :

$$
A M R=\frac{1}{N} \sum_{i=1}^{N} \frac{P_{i}^{2}}{P_{i}^{1}}=\frac{1}{N} \sum_{i=1}^{N} a_{i}=\bar{a}
$$

This price index is used by Ashenfelter (1989), and McAfee and Vincent (1993), in studies dealing with similar lots of wine. Both find that the mean of the ratios is significantly less than one for each of the auction houses, and conclude in favor of a price decline.

The Ratio of the Sums of Prices is the sum of the second prices divided by the sum of the first prices. It aggregates first prices and second prices, and can be written as:

$$
R S P=\frac{\sum_{i=1}^{N} P_{i}^{2}}{\sum_{i=1}^{N} P_{i}^{1}}=\frac{\sum_{i=1}^{N} a_{i} P_{i}^{1}}{\sum_{i=1}^{N} P_{i}^{1}}
$$

Pesando and Shum (1996) use the RSP method and find a price decline for lots of identical Picasso prints. This method weights individual ratios $a_{i}$ differently according to the level of $P_{i}^{1}$, and computes price variations in a way that is closely related to the calculation of the yield of a financial portfolio, where the number of each type of share is weighted by its individual yield.

The spread between RSP and AMR depends on the covariance and the mean of the first prices (see appendix for details) :

$$
R S P-A M R \simeq \frac{\operatorname{cov}\left(a, P^{1}\right)}{\bar{P}^{1}}
$$

where $\operatorname{cov}\left(a, P^{1}\right)$ is the covariance between $a_{i}$ and $P_{i}^{1}$, and $\bar{P}^{1}=\frac{1}{N} \sum_{i=1}^{N} P_{i}^{1}$.

The axiomatic approach to index number theory (see Diewert (1987)) identifies properties that an index should verify. The relevant properties for 
bilateral indices and unit quantity are : identity, proportionality, monotonicity, invariance to a change in scale, in units of measurement, symmetrical treatment of time and mean value. The AMR does not satisfy the "time reversal" property assuming symmetrical treatment of time, which is certainly a serious flaw for a price index. Assume that two pairs of objects are sold at prices of 10 and 20 for the first pair, and 20 and 10 for the second. The arithmetic mean of the ratios is 1.25 , an increase of $25 \%$, although there is actually no price increase. ${ }^{6}$ The RSP does not satisfy the "invariance to change in units of measurement" property if this change only affects some of the objects sold.

\subsection{Two alternative indices}

Let us consider two other indices, the Geometric Mean of Ratios (GMR) and the Fisher Price Index (FPI). They verify all seven properties. However, neither the GMR nor the FPI has apparently been used in the literature to aggregate price movements in auctions. The Geometric Mean of Ratios can be written as :

$$
G M R=\left[\prod_{i=1}^{N}\left(\frac{P_{i}^{2}}{P_{i}^{1}}\right)\right]^{\frac{1}{N}}=\left[\prod_{i=1}^{N} a_{i}\right]^{\frac{1}{N}}
$$

where $\operatorname{var}(a)$ is the variance of $a$. This method requires every price to be strictly positive, which is clearly the case with auction prices.

Using a second-order Taylor approximation, the difference between GMR and AMR appears to be :

$$
G M R-A M R \simeq A M R \times\left[\exp \left(\frac{-0.5 \operatorname{var}(a)}{A M R^{2}}\right)-1\right]
$$

The Fisher Price Index often appears in the index number literature as an ideal choice. It is defined as:

$$
F P I=\left[\frac{\sum_{i=1}^{N} a_{i}}{\sum_{i=1}^{N} \frac{1}{a_{i}}}\right]^{\frac{1}{2}}=[A M R \times H M R]^{\frac{1}{2}}
$$

where $H M R=N / \sum_{i=1}^{N} 1 / a_{i}$. Using a second order Taylor approximation, we have :

$$
F P I-A M R \simeq \frac{-0.5 \operatorname{var}(a)}{A M R}
$$

6 This drawback is known in the literature as çcomputationformula biasç (see Diewert (1998), and Reinsdorf (1998)). According to the Boskin Commission (1996), it helps to explain the overestimation of the US Consumer Price Index. 
An expression of the spread between FPI and GMR is given by :

$$
F P I-G M R=A M R\left[1-\frac{0.5 \operatorname{var}(a)}{(A M R)^{2}}-\exp \frac{(-0.5 \operatorname{var}(a))}{(A M R)^{2}}\right]
$$

Analytical expressions of the spreads make it possible to rank the four price indices, given that $P_{i}^{1}$ and $P_{i}^{2}$ are strictly positive. Hence, $\operatorname{var}(a)=0$ implies AMR $=$ FPI $=$ GMR $=\mathrm{RSP}$, whereas $\operatorname{var}(a) \neq 0$ implies FPI $<$ GMR $<$ AMR. The ranking of RSP depends on $\operatorname{cov}\left(a, P^{1}\right):$ if $\operatorname{cov}\left(a, P^{1}\right)>0, \mathrm{AMR}<\mathrm{RSP}$, if $\operatorname{cov}\left(a, P^{\mathrm{l}}\right)<\frac{-0.5 v a r(a) \overline{P^{1}}}{(A M R)^{2}}<0, \mathrm{RSP}<\mathrm{FPI}$, else we have $\mathrm{FPI}<\mathrm{RSP}<\mathrm{AMR}$.

To summarize, GMR and FPI are always very close, AMR is always larger than GMR and FPI, and AMR and RSP are very close only when $\operatorname{cov}\left(a, P^{1}\right)$ is close to zero. Hence, when $\operatorname{var}(a)$ is sufficiently large and/or $\operatorname{cov}\left(a, P^{1}\right)$ differs sufficiently from 0 , differences between the estimators may lead to different conclusions when applied to real data.

\section{Empirical evidence from wine auctions}

The data analyzed are 2,947 lots of wine sold at Christie's London between December 1995 and February 1996. ${ }^{7}$ A parcel is defined as a set of lots of similar wine of same vintage, same capacity, same lot size $(2,6,12,24, \ldots$ bottles) sold sequentially. Hence, there are 1,160 lots of wine belonging to 427 parcels, each of whose size is between 2 and 7 lots. $^{8}$ In section 3.1 , only the last two lots of each parcel are selected. This is how Ashenfelter (1989) and McAfee and Vincent (1993) proceeded, and the following sections explore whether the trend resulting from this choice is robust to methodological changes. The influence on the price trends of factors such as the analytical method used, the size of the parcel and the position of lots within the parcels is studied.

\subsection{Comparing the four estimators}

Prices between the last two lots of each parcel (referred to as sample 1) did not change in 282 cases (66\%), decreased in 127 cases $(29.7 \%$ ) and increased in 18 cases $(4.3 \%)$. The four indices, when computed for the 427 pairs of prices, confirm these first findings, each being negative, between $-1.53 \%$ for AMR and $-1.84 \%$ for RSP. They are denoted $\theta_{1}$ in Table 1.

\footnotetext{
7 We are very grateful to Victor Ginsburgh for allowing us to use his data.

8 The data consist of 244 percels of 2 lots, 104 parcels of 3 lots, 51 parcels of 4 lots, 15 parcels of 5 lots, 11 parcels of 6 lots and two parcels of 7 lots.
} 
Table 1 : Statistics for the four estimators for observed auction prices

\begin{tabular}{cccccc} 
& & AMR & GMR & RSP & FPI \\
\hline Original & $\theta_{1}$ (in \%) & -1.532 & -1.646 & -1.844 & -1.667 \\
Sample 1 & & & & & \\
\hline Bootstrap 1 & $\bar{\theta}_{1}^{*}$ (in \%) & -1.536 & -1.649 & -1.846 & -1.670 \\
sample & $\sigma_{1}^{*}\left(\times 10^{-2}\right)$ & 0.201 & 0.245 & 0.315 & 0.259 \\
(Last two & $95 \%$ conf. interval & $(-1.96,-1.17)$ & $-2.17,-1.22)$ & $(-2.51,-1.28)$ & $(-2.23,-1.22)$ \\
lots of & P-value for $\bar{\theta}_{1}^{*}=\theta_{1}$ & 0.973 & 0.951 & 0.967 & 0.942 \\
the parcel) & P-value for $\bar{\theta}_{1}^{*}=0$ & 0 & 0 & 0 & 0 \\
\hline Original & $\theta_{2}$ (in \%) & -2.320 & -2.475 & -2.697 & -2.506 \\
Sample 2 & & & & & \\
\hline Bootstrap 2 & $\bar{\theta}_{2}^{*}$ (in \%) & -2.319 & -2.473 & -2.698 & -2.504 \\
sample & $\sigma_{2}^{*}\left(\times 10^{-2}\right)$ & 0.234 & 0.285 & 0.427 & 0.305 \\
(First and & $95 \%$ conf. interval & $(-2.80,-1.89)$ & $(-3.08,-1.97)$ & $(-3.60,-1.92)$ & $(-3.16,-1.97)$ \\
last lots of & P-value for $\bar{\theta}_{2}^{*}=\theta_{2}$ & 0.971 & 0.950 & 0.954 & 0.940 \\
the parcel) & P-value for $\bar{\theta}_{2}^{*}=0$ & 0 & 0 & 0 & 0 \\
& P-value for $\bar{\theta}_{1}^{*}=\bar{\theta}_{2}^{*}$ & 0 & 0 & 0 & 0 \\
\hline
\end{tabular}

The 427 price pairs observed are considered to be drawn from a pair of unknown distribution functions, ${ }^{9}$ which will be used to study the empirical distributions and test the difference between the indices with bootstrap procedures.

427 pairs of prices were drawn with replacement from the 427 observed pairs. Then the four indices $\theta_{b}^{*}$ (AMR, GMR, RSP and FPI) were computed for these pairs. This was done $B$ times, and resulted in $B$ values $\theta_{b}^{*}=$ $\theta_{1}^{*}, \ldots, \theta_{B}^{*}$ for each of the indices. The empirical distribution for each $\theta^{*}$, its mean $\bar{\theta}^{*}$ and its variance $\left(\sigma^{*}\right)^{2}$ were also computed. It was then possible to observe their distribution and to test the spreads between methods. Bickel and Freedman (1981) showed, using Mallows distance, that the empirical distribution of $\theta^{*}$ is a convergent and unbiased estimator of the true distribution of $\theta$, if both the number of replications $B$ and the size $N$ of the original data are large.

The number of replications $B$ was set at 9,999 , which is large enough to allow standard error and confidence interval computations according to Efron and Tibshirani (1993). Results can be found in Table 1 for each of the four index estimators (bootstrap sample 1) : the mean $\bar{\theta}_{1}^{*}$, the standard deviation $\sigma_{1}^{*}$ and the $95 \%$ confidence interval of the mean based on the percentile distribution. The underlying distributions are represented in Figure 1 and confirm noticeable differences in location and dispersion among the four estimators.

Equality tests assume that the mean of an index estimator is equal to a given value under the null hypothesis. They are conducted on the

9 A joint normality test for each of the distributions points to log-normality at the $2.5 \%$ signiicance level. The mean and standard deviation are $\mathbf{4 2 0 . 5}$ and 484 for the irst price distribution, and 412.8 and 474 for the second distribution. 
Figuro 1 Bootstrap distribution for four relovant esttmators, 9,899 replications (sample 1)

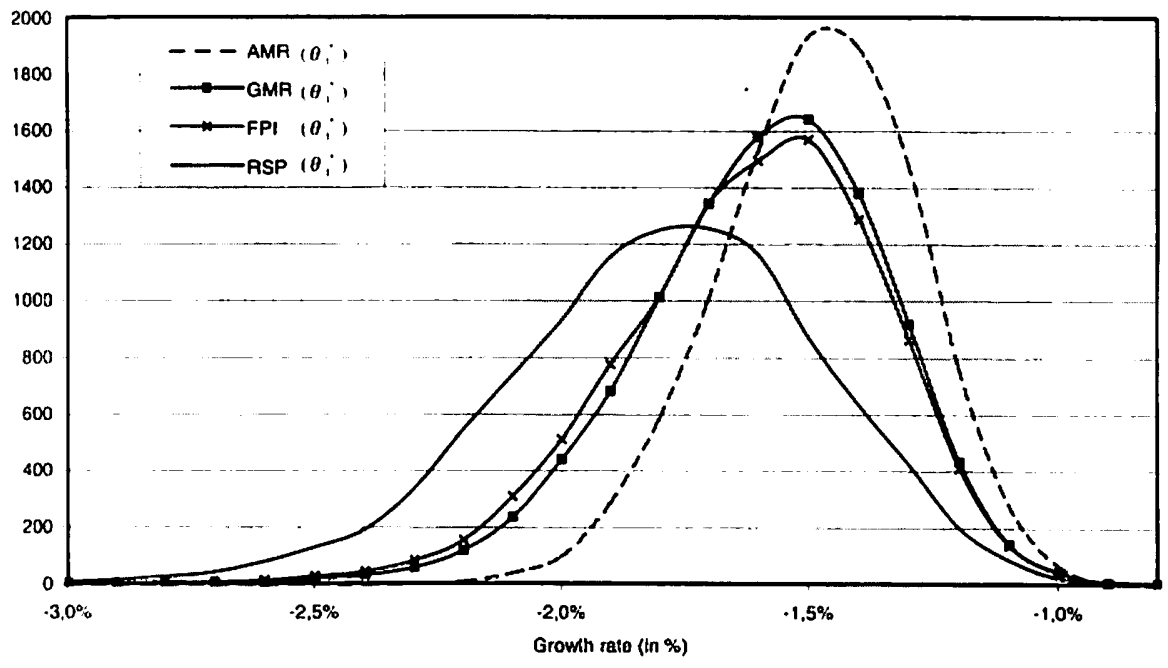

bootstrap percentile distribution for each index (see Table 1). Bilateral equality tests $\bar{\theta}_{1}^{*}=\theta_{1}$ showed that no method is significantly biased, and bilateral equality tests $\bar{\theta}_{1}^{*}=0$ confirmed a significant decline. The results obtained for each method are consistent and there is no doubt that prices decline in this sample. An important question is whether the four methods yield significantly different results on the magnitude of the price trend.

Equality tests between trends assume that the mean difference between two indices is zero under the null hypothesis. However, it would be meaningless to compare, two by two, the three indices linked by the strict inequality obtained in Section 2 (FPI $<$ GMR $<$ AMR). As a consequence, rather than testing that the mean difference is strictly equal to zero, the null hypothesis is that the difference between two indices is very small (less than $1 \%$ of the average index estimator, i.e. less than $0.0165 \%$ ). Each test uses the bootstrap percentile distribution of the difference between two indices as an estimator of the distribution of their differences. The null hypothesis is rejected for five tests out of $\operatorname{six}(\mathrm{p}$-value $<0.05)$ but not rejected for GMR-FPI ( $p$-value $=0.373$ ).

The equality tests between trends based on differences do not take into account the index distribution. Hence, the non-parametric distribution free Kolmogorov-Smirnov test is computed for each comparison. The null hypothesis is that the distribution of the two index estimators is similar. The maximum absolute difference between the cumulative distributions is computed and compared with the appropriate critical values (see for instance Daniel (1990)). The equality of the distributions for each comparison is very strongly rejected $(p$-value $<0.001$ ) except for GMR-FPI ( $p$-value $=0.338$ ). 
Figure 2 Cumulative Fraction Plots for Kolmogorov-Smirnov Test

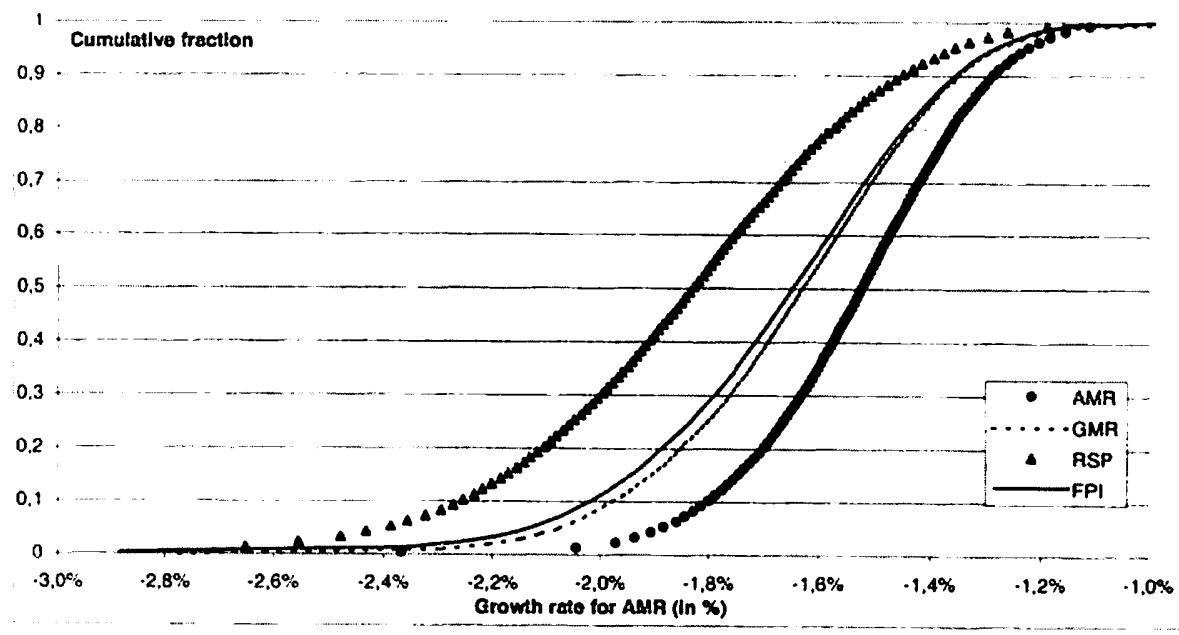

The bootstrap cumulative fraction plots corresponding to each index are shown in Figure 2 and confirm the closeness between GMR and FPI.

The fact that three index estimators out of four cannot be considered as having the same distribution suggests that caution is in order when the decline is not large.

\subsection{The importance of choosing pairs}

When a parcel contains more than two lots (this occurs for 183 out of 427 parcels in the data), there are different arbitrary ways of selecting a pair of prices. In order to study the sensitivity of the results to this choice, a second sample (referred to as sample 2) is constructed, in which the prices of the first and last lots of each of the 427 parcels, instead of the two last lots, are compared. It should be noted that only the first price of each pair was different from the previous sample.

The bootstrap distribution of the estimators is computed for these new pairs and the statistics are also given in the lower part of Table 1. The decline is larger than above and significant for each method ( $\mathrm{p}$-value $=0$ for all $\bar{\theta}_{2}^{*}=0$ tests). The difference between bootstrap estimators $\bar{\theta}_{1}^{*}$ and $\bar{\theta}_{2}^{*}$ - based on the bootstrap percentile distribution of their difference - is highly significant for each method ( $p$-values $<0.001$ ), which indicates that the position of a lot in (and/or the size of) a parcel is a relevant piece of information. This result is consistent with van den Berg et al. (2001) who observe in flower auctions that the fewer the remaining number of lots in a parcel, the larger the price decline. 
Table 2 : Influence of lot position in each parcel

\begin{tabular}{cccccc|c} 
Lot position in the parcel & $1-2$ & $2-3$ & $3-4$ & $4-5$ & $5-6 \&$ 6-7 & All \\
\hline No. of pairs & 427 & 183 & 79 & 28 & 15 & 732 \\
\hline AMR (in \%) & -1.642 & -1.265 & -0.682 & -0.598 & 0.160 & -1.367 \\
P-value of nullity test & 0 & 0 & 0.028 & 0.011 & 0.762 & 0 \\
GMR (in \%) & -1.704 & -1.424 & -0.735 & -0.606 & 0.147 & -1.450 \\
P-value of nullity test & 0 & 0 & 0.022 & 0.012 & 0.764 & 0 \\
RSP (in \%) & -1.959 & -1.569 & -0.869 & -0.456 & 0.343 & -1.656 \\
P-value of nullity test & 0 & 0 & 0.019 & 0.012 & 0.554 & 0 \\
FPI (in \%) & -1.706 & -1.469 & -0.737 & -0.606 & 0.147 & -1.463 \\
P-value of nullity test & 0 & 0 & 0.023 & 0.012 & 0.764 & 0 \\
\hline
\end{tabular}

Marginal significance levels ( $p$-values) are computed from the bootstrap distributions.

The price effects specific to the position of the lots in the parcel are detailed in Table 2.

The average decline between two successive pairs varies from -1.37 to $-1.66 \%$. For all the methods, there is a significant price decline between lots 1 and 2 ( -1.64 to $-1.96 \%$, p-value close to 0$)$. The additional decline between lots 2 and 3 is smaller but also significant ( -1.27 to $1.57 \%)$. For the next lots (3 and 4,4 and 5), the decline is even smaller and significant at the $5 \%$ level. These results confirm the importance of the choice of pairs when computing price indices.

In fact, this is the result of two factors : a "position in the parcel" effect and a "size of the parcel" effect. ${ }^{10}$ Figure 3 represents the effect of the position of the pair depending on the parcels' size for AMR (results are almost identical for the other estimators). For a given size, the price decline between two successive lots is generally smaller when lots are positioned higher. An overall increase is observed when parcels of different sizes are pooled.

Figure 4 represents the effect of parcel size depending on the position of the pair. The price decline between two successive lots is generally smaller when parcel size increases, although there are differences depending on position in the parcel. The larger the size of the parcel, the smaller the average price decline between the successive lots of this parcel. The size effect is weaker than the position effect but both are significant when separately estimated in a regression equation. ${ }^{11}$

Parcel size therefore constitutes relevant information when studying price trends in sequential auctions. The fact that prices decline the lower the

10 We are grateful to an anonymous referee that drew our attention to this point.

11 The strong correlation that obviously exists between these two effects certainly explains why a regression analysis does not give signilcant results when both effects are included. Van den Berg ot al. (2001), when studying the effect of the position of the lot and the remaining number of lots (see their Table 3), in fact measure a joint çsize-positioné effect and a joint çsize-remaining number of lotse effect. As they are only interested in comparing the position effect and the çremaining number of lotse effect, their conclusions remain valid. 

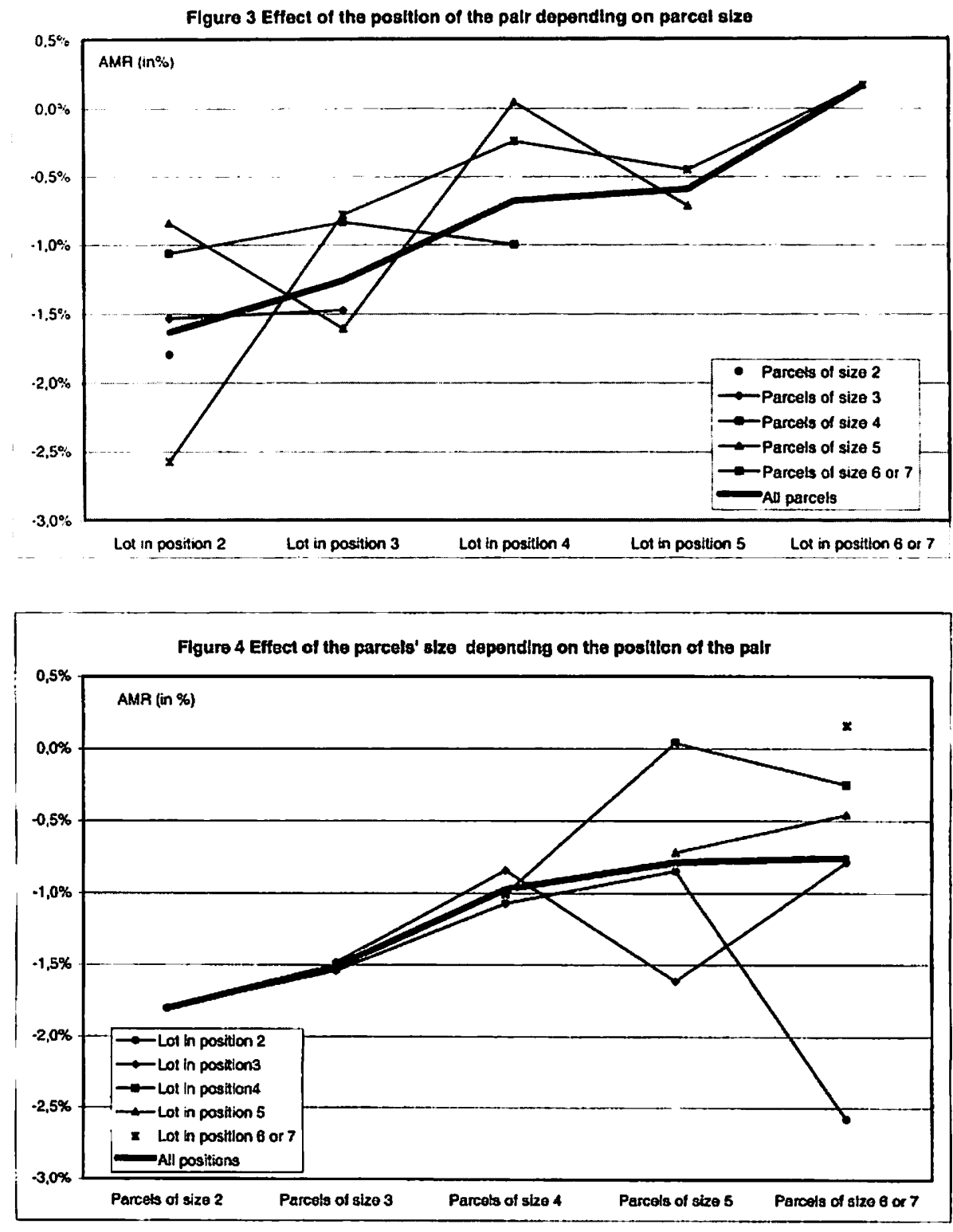

position of lots within a parcel probably results from stronger competition between tidders for the first lots. This competition may result from a supply uncertainty due to the presence of written bids and the existence of a buyer's option that allows the winner of the first lot in a parcel to buy any number of similar lots from the parcel at the same price. Indeed, oral bidders know that the first lot of each parcel is the only one they are certain to compete for. 


\subsection{Does the direction of the trend depend on the way it is computed?}

As shown previously, different price trends can be obtained by choosing different pairs when using the same method. It is interesting to determine whether the way the price trend is computed may also influence its direction (upward or downward). However, the price trends established on the previous wine data show a very significant decline, and findings regarding their direction did not change depending on the method or the criterion chosen for selecting pairs. Moreover, identical objects generally imply a rather small variance for $a$, and Section 2 shows that the four methods lead to similar results in this situation. Yet it is possible to use the data to mimic a weak trend. The price vector $P^{2}$ is thus set to $\tilde{P}^{2}=(0.98)^{-1} P^{2}$ in order to shift the distribution of each of the four estimators to the right. The corresponding distributions $\tilde{\theta}_{1}$ (last two lots) and $\tilde{\theta}_{2}$ (first and last lots) of each estimator are simulated by bootstrapping. Unilateral equality tests $\tilde{\theta}_{1}=0$ and $\tilde{\theta}_{2}=0$ are then conducted. If equality is confirmed, there is no trend and prices are stable. If equality is not confirmed, the sign of the estimator determines the direction of the trend (increase or decrease), and the bootstrap percentile p-value gives the level of significance.

The results are given in Table 3 and can be interpreted as follows. For each of the four indices, the result of the tests $\tilde{\theta}_{1}=0$ and $\tilde{\theta}_{2}=0$ is represented in boldface type in a column and in italic type in a row, respectively. Each cell checks whether the two ways of selecting pairs lead to identical conclusions for the same method on the diagonal, or for different methods off the diagonal.

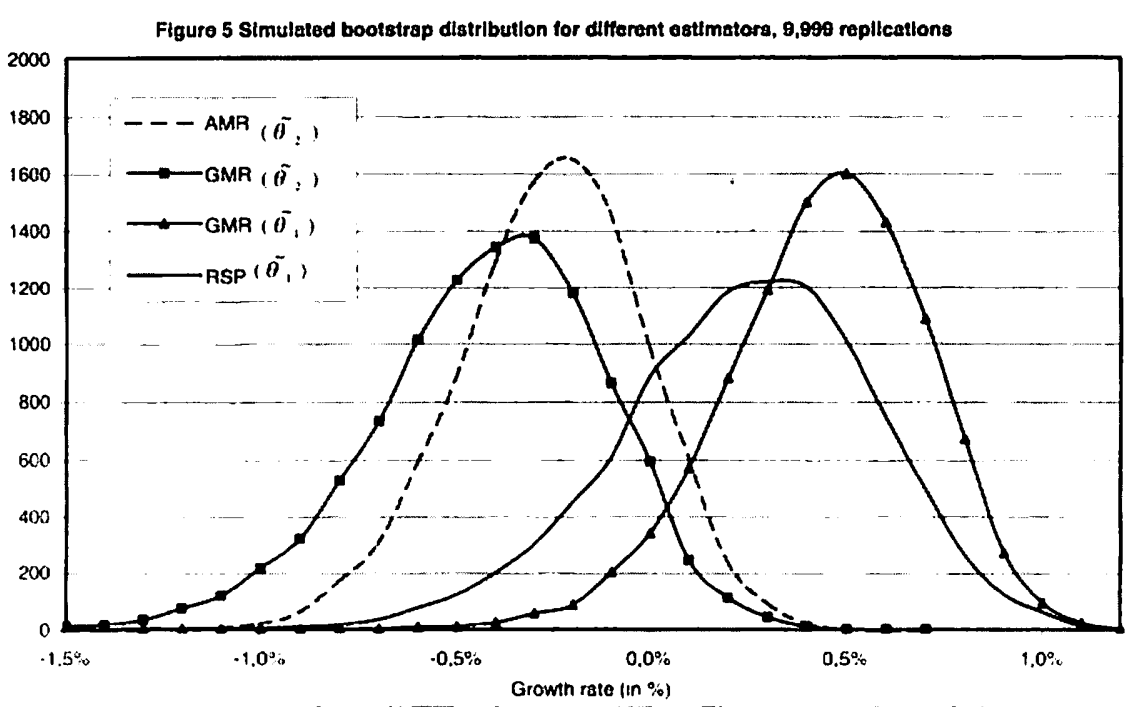


Table 3: Conclusion of equality test $\tilde{\theta}_{1}=0$ and $\tilde{\theta}_{2}=0$

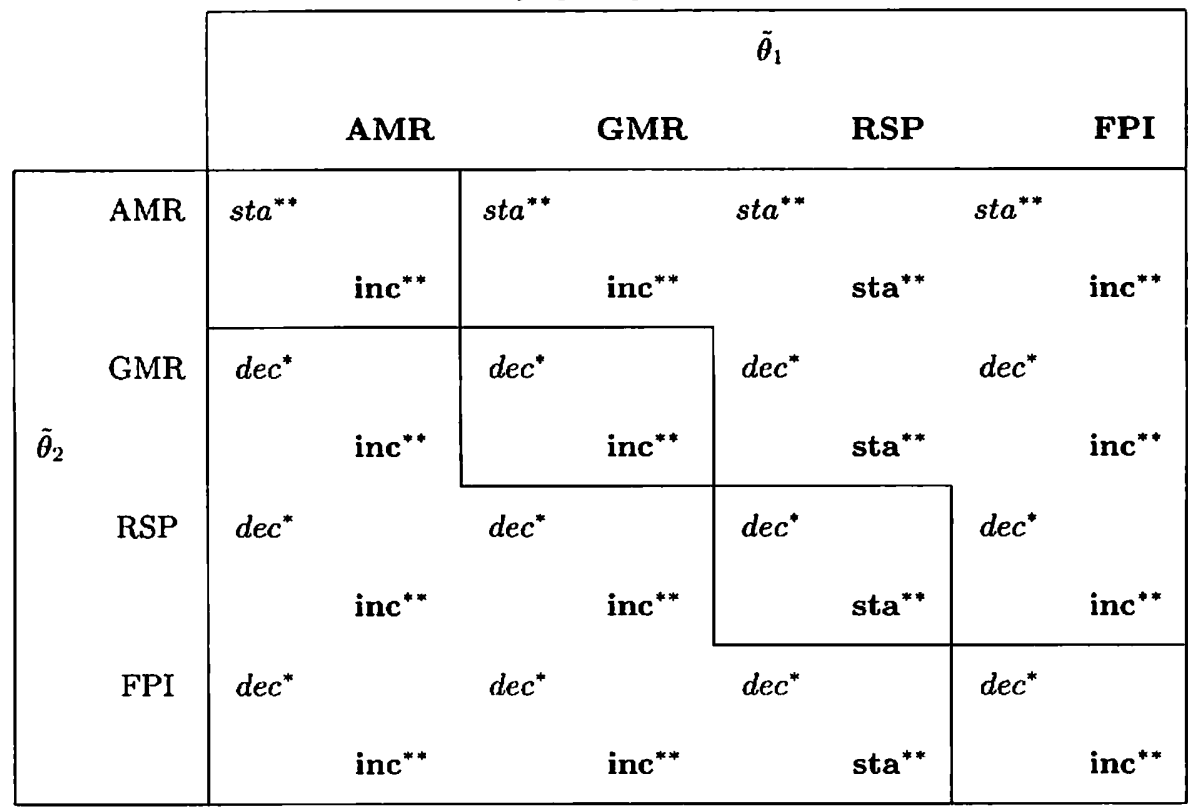

For each estimator, the conclusion of equality test $\tilde{\theta}_{1}=0$ is in boldface and $\tilde{\theta}_{2}=0$ is in italic. dec for decrease, inc for increase and sta for stability.

${ }^{*}$ : p-value $<0.10{ }^{* *}$ : p-value $<0.05$.

Analyzing Table 3 by column (or by row) shows that the direction of the price trend computed with RSP (or with AMR) differs from the one obtained by the other three methods. The diagonal shows that the same method applied with different criteria for extraction from the parcels leads to different conclusions, and even to opposite conclusions with GMR and FPI (increase vs. decrease). Finally, different methods with different extraction criteria lead to the same conclusion in only one case out of 12 , to different conclusions in four cases and to opposite conclusions in the remaining seven cases!

Figure 5 illustrates two of the most puzzling results. First, the distribution of RSP in sample $1, \tilde{\theta}_{1}$, (or AMR in sample $2, \tilde{\theta}_{2}$ ) is not significantly different from zero whereas the three other index estimators are increasing (or decreasing). Second, the GMR distributions in both samples, $\tilde{\theta}_{1}$ and $\tilde{\theta}_{2}$, show substantial differences, which confirms the previous conclusion that the trends are opposite (as seen in Table 3 ).

Hence, in the case of weak trends, two methods, even based on the same extraction criterion, may lead to different conclusions. 


\section{Conclusion}

The aim of this paper was to assess the sensitivity of the measurement of price trends in sequential auctions to methodological choices. We purposely consider the objects sold as identical, and study the properties and the dispersion of four common price indices in this framework. Wine auction data are used to compute (and simulate) their spread and to test various hypotheses.

The main conclusion is that the choice of a method to measure the price trend is problematic. Different methods may lead to different results when applied to real auction prices, in particular with weak trends. In order to limit specific bias, it is certainly worth computing three index numbers : AMR, RSP and either GMR or FPI (considering their respective distributions are not significantly different) to determine and quantify price trends. The choice of the pairs of objects, the size of the parcels and the position of the lots in each parcel also influence the results.

Lastly, bearing in mind that similar objects are not strictly identical and that some differences may only be observed by bidders attending the auction, thereby influencing their valuations, conventional approaches used to study price movements for homogeneous goods may not be correct. When these differences are known, a better approach may be to first run hedonic regressions explaining prices by reference to objective characteristics of the objects (including quality differences), and then to compute the different measurements of the price trend, corrected for quality differences.

\section{Appendix}

\section{Notation}

Consider a sample $a_{i}, \ldots, a_{N}$ an iid $\tilde{a}$ where $\tilde{a}$ is a positive random variable with finite variance.

Let us define the following quantities :

$$
A M R=\frac{1}{N} \sum_{i=1}^{N} a_{i}=\bar{a} \simeq E\left[a_{i}\right], \quad i=1, \ldots, N,
$$

by the law of large numbers.

$$
\begin{gathered}
H M R=N \sum_{i=1}^{N} a_{i}^{-1}, \\
G M R=\left[\prod_{i=1}^{N} a_{i}\right]^{\frac{1}{N}},
\end{gathered}
$$




$$
\begin{gathered}
F P I=[A M R \times H M R]^{\frac{1}{2}}, \\
R S P=\frac{\sum_{i=1}^{N} P_{i}^{2}}{\sum_{i=1}^{N} P_{i}^{1}} \simeq \frac{E\left[P_{i}^{2}\right]}{E\left[P_{i}^{1}\right]}, \quad i=1, \ldots, N,
\end{gathered}
$$

It is recalled that a second-order Taylor series approximation of $f\left(a_{i}\right)$ around $E\left[a_{i}\right]$ is :

$$
f\left(a_{i}\right) \simeq f\left[E\left(a_{i}\right)\right]+f^{\prime}\left[E\left(a_{i}\right)\right] \times\left[a_{i}-E\left(a_{i}\right)\right]+\frac{1}{2} f^{\prime \prime}\left[E\left(a_{i}\right)\right] \times\left[a_{i}-E\left(a_{i}\right)\right]^{2} .
$$

Hence, $E\left[f\left(a_{i}\right)\right]$ is equal to:

$$
E\left[f\left(a_{i}\right)\right] \simeq f\left[E\left(a_{i}\right)\right]+\frac{1}{2} f^{\prime \prime}\left[E\left(a_{i}\right)\right] \times \operatorname{var}\left(a_{i}\right),
$$

where $\operatorname{var}\left(a_{i}\right)$ is the variance of $a_{i}, i=1, \ldots, N$.

\section{Approximation of the bias between AMR and RSP}

Rewrite $E\left(P_{i}^{2}\right)$ as :

$$
E\left(P_{i}^{2}\right)=E\left(a_{i} P_{i}^{1}\right)=E\left(a_{i}\right) E\left(P_{i}^{1}\right)+\operatorname{cov}\left(a_{i}, P_{i}^{1}\right) \quad i=1, \ldots, N,
$$

where $\operatorname{cov}(\cdot)$ denotes the covariance.

Hence, by (13) :

$$
E\left(a_{i}\right)+\frac{\operatorname{cov}\left(a_{i}, P_{i}^{1}\right)}{E\left(P_{i}^{1}\right)}=\frac{E\left(P_{i}^{2}\right)}{E\left(P_{i}^{1}\right)} \simeq R S P \quad i=1, \ldots, N .
$$

\section{Approximation of the bias between AMR and GMR}

Note that $\ln (G M R)=(1 / N) \sum_{i=1}^{N^{\prime}} \ln \left(a_{i}\right)$ and that

$\ln (A M R)=\ln \left[(1 / N) \sum_{i=1}^{N} a_{i}\right]$ the logarithm function being concave, Jensen's inequality yields :

$$
\ln (A M R) \geqslant \ln (G M R) .
$$

Hence,

$$
\ln (A M R)-\ln (G M R) \geqslant 0 .
$$

Taking a second-order Taylor series approximation of $\ln \left(a_{i}\right)$ around $E\left(a_{i}\right)$, and from (14), we obtain :

$$
E\left[\ln \left(a_{i}\right)\right] \simeq \ln \left[E\left(a_{i}\right)\right]-\frac{1}{2} \times \frac{\operatorname{var}\left(a_{i}\right)}{\left[E\left(a_{i}\right)\right]^{2}}
$$

Hence,

$$
\ln (A M R)-\ln (G M R) \simeq \frac{1}{2} \times \frac{\operatorname{var}\left(a_{i}\right)}{\left[E\left(a_{i}\right)\right]^{2}}
$$


From there, the exponential yields :

$$
G M R \simeq A M R \times \exp \left(\frac{-0.5 \operatorname{var}\left(a_{i}\right)}{A M R^{2}}\right)
$$

\section{Approximation of the bias between FPI and AMR}

The approximation of the substitution bias formula for the CPI (see Diewert (1998)) is used and adapted to the bilateral framework.

Let $\varepsilon$ be the random variable defined by the values:

$$
\varepsilon_{i} \equiv \frac{a_{i}}{E\left(a_{i}\right)} \quad i=1, \ldots, N
$$

Note that

$$
E(\varepsilon)=\frac{1}{N} \sum_{i=1}^{N} \varepsilon_{i} \equiv 1 .
$$

Moreover, according to (10), $H M R$ may be written as :

$$
H M R=E\left(a_{i}\right) \times\left[\frac{1}{N} \sum_{i=1}^{N} \varepsilon_{i}^{-1}\right]^{-1} .
$$

Taking a second-order Taylor series approximation of $\varepsilon_{i}^{-1}$ around $\varepsilon_{i}=1$, and according to (14) :

$$
E\left(\varepsilon_{i}^{-1}\right) \simeq 1+\operatorname{var}\left(\varepsilon_{i}\right) .
$$

In order to obtain the inverse development for $E\left(\varepsilon_{i}^{-1}\right)$, the coefficients in the identity $E\left(\varepsilon_{i}^{-1}\right) \times\left[E\left(\varepsilon_{i}^{-1}\right)\right]^{-1} \equiv 1$ are characterized and after some computation, it follows that :

$$
\left[E\left(\varepsilon_{i}^{-1}\right)\right]^{-1} \simeq 1-\operatorname{var}\left(\varepsilon_{i}\right) .
$$

Substituting HMR in (12), leads to:

$$
F P I \simeq E\left(a_{i}\right) \times\left[1-\operatorname{var}\left(\varepsilon_{i}\right)\right]^{\frac{1}{2}} .
$$

Taking the second-order Taylor series approximation of $\operatorname{var}\left(\varepsilon_{i}\right)$ around 0 yields :

$$
F P I \simeq E\left(a_{i}\right) \times\left[1-0.5 \operatorname{var}\left(\varepsilon_{i}\right)\right] .
$$

Hence, from (16) and (9):

$$
F P I \simeq A M R \times\left[1-\frac{0.5 v a r\left(a_{i}\right)}{A M R^{2}}\right]
$$




\section{Approximation of the bias between FPI and GMR}

Using (15) and (17):

$$
F P I \simeq G M R \times \exp \left[\frac{0.5 v a r\left(a_{i}\right)}{A M R^{2}}\right] \times\left[1-\frac{0.5 v a r\left(a_{i}\right)}{A M R^{2}}\right]
$$

Q.E.D.

\section{References}

Ashenfelter O. (1989), "How auctions work for wine and art", Journal of Economic Perspectives, 3, pp. 23-36.

Ashenfelter O. and D. Genesove (1992), "Testing for price anomalies in real-estate auctions", American Economic Review, 82, pp. 501-505.

Beggs A. and K. Graddy (1997), "Declining values and the afternoon effect : Evidence from art auctions", RAND Journal of Economics, 28, pp. 544-565.

Bickel P.J. and D.A. Freedman (1981), "Some asymptotic theory for the bootstrap", Annals of Statistics, 9, pp. 1196-1217.

Boskin M. (1996), "Towards a more accurate measure of the cost of living", Report to the Senate finance committee by the Advisory Commission to study the consumer price index, Dec. 5 .

Buccola S. (1982), "Price trends at livestock auctions", American Journal of Agricultural Economics, 64, pp. 63-69.

Chanel O., L-A. Gérard-Varet and S. Vincent (1996), "Auction theory and practice: Evidence from the market of jewellery", in V. Ginsburgh and P.-M. Menger (eds.), Economics of the arts: Selected essays, Amsterdam, North Holland, pp. 135-149.

Daniel W. (1990), Applied nonparametric statistics, Second edition, Boston, PWS-Kent.

Di Vittorio A. and V. Ginsburgh (1994), "Pricing red wines of Médoc vintages from 1949 to 1989 at Christie's auctions", Mimeo, University Libre de Bruxelles, Bruxelles.

Diewert W.E. (1987), "Index numbers", in J. Eatwell, M. Milgate and P. Newman, (eds.), The new Palgrave: A dictionary of economics, London, The Macmillian Press, pp. 767-780.

Diewert W.E. (1998), "Index number issues in the consumer price index", Jiurnal of Economic Perspectives, 12, pp. 47-58.

Efron B. and R.J. Tibshirani (1993), "An introduction to the bootstrap", Monographs on statistics and applied probability, 57, New York and London, Chapman and Hall. 
Fevrier P. (2003), "Enchères multi-unités : une approche théorique, empirique et expérimentale", unpublished $\mathrm{PhD}$ Dissertation, École des Hautes Études en Sciences Sociales, Paris.

Ginsburgh V. (1998), "Absentee bidders and the declining price anomaly in wine auctions", Journal of Political Economy, 106, pp. 1302-1319.

Lusht K. (1994), "Order and price in a sequential auction", Journal of Real Finance and Economics, 8, pp. 259-266.

McAfee R.P. and D. Vincent (1993), "The declining price anomaly", Journal of Economic Theory, 60, pp. 191-212.

Milgrom P. and R.J. Weber (2000), "A theory of auctions and competitive bidding II", in P. Klemperer (ed.), The economic theory of auctions, Cheltenham, Edward Elgar.

Pesando J.E. and P.M. Shum (1996), "Price anomalies at auction : Evidence from the market for modern prints", in V. Ginsburgh and P.-M. Menger (eds.), Economics of the arts : Selected essays, Amsterdam, North Holland, pp. 113-134.

Pezanis-Christou P. (1997), "Three essays on competitive bidding", unpublished PhD Dissertation, European University Institute, Florence.

Reinsdorf M.B. (1998), "Formula bias and within-stratum substitution bias in the U.S. CPI", The Review of Economics and Statistics, 80, pp. 175187.

Taylor W.M. (1991), "Declining prices in sequential auctions : An empirical investigation", Working Paper, ${ }^{\circ} 90$, Rice University, Houston.

Van Den Berg G.J., J.C. Van Ours and M.P. Pradhan (2001), "The declining price anomaly in Dutch Dutch rose auctions", American Economic Review, 91 : 4, pp. 1055-1062.

Weber R.J. (1983), "Multiple-object-auctions", in R. Engelbrecht-Wiggans, M. Shubik and R.M. Stark (eds.), Auctions, bidding, and contracting: Uses and theory, New York, New York University Press. 